Audiology

Neurotology
Audiol Neurotol 2018;23:229-237

DOI: $10.1159 / 000494021$
Received: June 12, 2018

Accepted after revision: September 21, 2018

Published online: November 15, 2018

\title{
Visual Analogue Scales as a Tool for Initial Assessment of Tinnitus Severity: Psychometric Evaluation in a Clinical Population
}

\author{
Danuta Raj-Koziak ${ }^{a} \quad$ Elzbieta Gos ${ }^{a} \quad$ Weronika Swierniak ${ }^{a}$ Joanna J. Rajchel ${ }^{a}$ \\ Lucyna Karpiesz $^{a} \quad$ Iwona Niedzialek ${ }^{a}$ Elzbieta Wlodarczyk ${ }^{a}$ Henryk Skarzynskia \\ Piotr H. Skarzynski ${ }^{\mathrm{a}-\mathrm{c}}$ \\ aWorld Hearing Center, Institute of Physiology and Pathology of Hearing, Warsaw/Kajetany, Poland; \\ ${ }^{b}$ Heart Failure and Cardiac Rehabilitation Department, Medical University of Warsaw, Warsaw, Poland; \\ 'Institute of Sensory Organs, Warsaw/Kajetany, Poland
}

\section{Keywords}

Tinnitus · Visual analog scale · Validity · Reliability

\begin{abstract}
The aim of this study was to evaluate the psychometric properties of patient-reported visual analogue scale (VAS) ratings. All of the participants (100 Polish-speaking adults) completed a Tinnitus Functional Index (TFI) once and a 4-component VAS twice over a period of 3 days. Spearman's correlation coefficients between the VAS score and global TFI ranged from $\rho=0.52$ for VAS-coping (VAS-C) to $\rho=0.81$ for VAS-annoyance (VAS-A). Using the Bland-Altman method, the agreement ranged from $93 \%$ for VAS-A to $96 \%$ for VAS-distress (VAS-D). Interclass correlation coefficients ranged from 0.67 for VAS-C to 0.90 for VAS-A. The VAS cutoff points representing significant tinnitus severity ranged from 45 points for VAS-C to 66 points for VAS-D. VAS scales are a valid and reliable brief screening tool for obtaining quick information about tinnitus.

(c) 2018 The Author(s) Published by S. Karger AG, Basel
\end{abstract}

\begin{tabular}{ll}
\hline KARGER & ( 2018 The Author(s) \\
& Published by S. Karger AG, Basel Oparger \\
E-Mail karger@karger.com & This article is licensed under the Creative Commons Attribution- \\
www.karger.com/aud & $\begin{array}{l}\text { NonCommercial-NoDerivatives 4.0 International License (CC BY- } \\
\text { NC-ND) (http://www.karger.com/Services/OpenAccessLicense). } \\
\text { Usage and distribution for commercial purposes as well as any dis- } \\
\text { tribution of modified material requires written permission. }\end{array}$
\end{tabular}

\section{Introduction}

After analyzing the prevalence of tinnitus over several decades, the general conclusion is that it increases with age. The prevalence tends to increase from $4-6 \%$ in young adults to $10-20 \%$ in those aged $70-79$ years [Axelsson and Ringdahl, 1989; Ries, 1994; Davis and MRC Institute of Hearing Research, 1995; Shargorodsky et al., 2010; Fabijańska et al., 2012; McCormack et al., 2014; Kim et al., 2015; Martinez et al., 2015].

There are 2 main kinds of tinnitus, i.e. subjective and objective, with the first being the most common. The difficulty with subjective tinnitus is that there are no objective measures, and so the patient's description of their symptoms is the only source of information. The severity of tinnitus does not seem to be related to age [McCormack et al., 2014]. To decide whether a tinnitus-specific intervention is called for, in addition to taking a detailed case history and conducting otological and audiological assessments, validated tinnitus questionnaires can be used to assess the levels of tinnitus-related distress and tinnitus handicap and the patient's emotional reactions. According to Baguley et al. [2016], the 2 most frequently

Piotr H. Skarzynsk

Mokra 17 Street

PL-05-830 Nadarzyn (Poland)

E-Mail p.skarzynski@ifps.org.pl 
used questionnaires in the world are the Tinnitus Handicap Inventory (THI) and the Tinnitus Functional Index (TFI). These questionnaires are helpful tools for quantifying the disabling and handicapping effects of tinnitus. The questionnaires can gauge negative reactions associated with tinnitus, such as difficulty sleeping, concentration problems, anxiety, and annoyance.

During the tinnitus patient's first visit to the clinic, the clinician concentrates on identifying underlying causes that might be amenable to surgical or medical treatment. At this initial visit, when tinnitus is a symptom to be diagnosed rather than a condition to be treated, there would be value in classifying tinnitus severity quickly and reliably without having to fill in questionnaires. Such a type of screening tool might, we suggest, be the Visual Analog Scale (VAS). A VAS is a rating scale in which the subject rates their health outcomes subjectively and places a corresponding mark along a printed line [Torrance et al., 2001]. We see VAS as a tool that could allow the clinician to quickly gauge the loudness of tinnitus, its annoyance, the distress it is causing, and how well the patient is coping with the condition. Such a screening tool would allow the level of tinnitus perceived by the patient and their reaction to it to be estimated based solely on a few questions - although, of course, a detailed quantitative assessment of tinnitus severity would still require the available questionnaires [Langguth et al., 2011].

The purpose of this study was to evaluate the psychometric properties of 4 patient-reported VAS designed to gauge subjectively perceived levels of tinnitus loudness, annoyance, and distress and how well the patient is coping.

\section{Materials and Methods}

Validation was conducted in a clinical population of consecutive patients who were hospitalized for 3 days to fully diagnose their tinnitus (a standard procedure for tinnitus treatment in Poland). Exactly 100 adult patients (47 women and 53 men) underwent evaluation of their tinnitus at the Audiology and Phoniatrics Clinic and signed an informed consent form. During this diagnostic period no therapeutic procedures were applied. This trial was approved by the Ethics Committee of IFPH (IFPS: KB/10/2015) and complied with the Declaration of Helsinki.

Participants ranged in age from 19 to 75 years (mean $=51.96$, $\mathrm{SD}=13.62)$. All reported chronic tinnitus with a mean duration ranging from 0.25 to 40 years (mean $=7.16, \mathrm{SD}=7.34$ ). The most frequently reported type of tinnitus was bilateral (49\%); $34 \%$ of the participants reported unilateral tinnitus (in the left ear, 24\%; in the right ear, 10\%), and $17 \%$ heard tinnitus in the head.

A Polish version of a 4-component VAS measuring tinnitus loudness (VAS-L), annoyance (VAS-A), distress (VAS-D), and
VAS-L

1. How loud was your tinnitus over the last week?

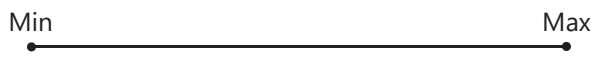

VAS-A

2. How much did your tinnitus annoy you over the last week?

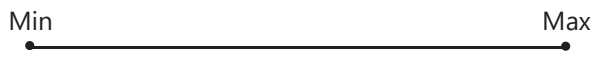

VAS-D

3. How much did your tinnitus distress you over the last week?

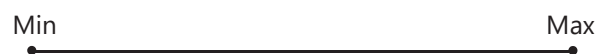

VAS-C

4. How well did you cope with your tinnitus over the last week?

I coped with

tinnitus problem

I did not cope with tinnitus at all

Fig. 1. VAS survey form for VAS-L, VAS-A, VAS-D, and VAS-C. The original form was in Polish, and it was filled out by Polishspeaking patients; this is an English translation.

how well the patient was coping with the problem (VAS-C) was created by a group of experts including clinicians, otolaryngologists, audiologists and psychologists, each with years of experience in managing tinnitus patients. An English translation of the VAS form used is shown in Figure 1.

The purpose and use of the VAS were explained to the patients. They were informed that the scales were designed to assess the loudness of their tinnitus, its annoyance, the degree of distress it was causing, and how well they were coping with it. The idea of a VAS is that it gauges a subjective reaction based on a line with defined end points. For example, the end points for measuring tinnitus loudness (VAS-L) are set as "inaudible" at one end and "extremely loud" at the other. For tinnitus annoyance (VAS-A), the end points are designated "not annoying" and "extremely annoying." Similarly, tinnitus distress (VAS-D) uses the end points "not distressing" and "extremely distressing." Finally, to measure how well the patient is coping with their tinnitus, the VAS-C scale ranges from "coping with tinnitus without problems" to "not coping with tinnitus at all” (Fig. 1).

The ratings of inaudible, not annoying, not distressing, and coping with tinnitus without problems occupied the left side of the form, and the opposite situation, where the tinnitus was rated as extremely loud, extremely annoying, extremely distressing, and not coping with tinnitus at all, occupied the right side of the form. Patients were asked to put a mark on the line (100 mm long) between these extremes to represent how strongly they rated their tinnitus. The mark was usually placed somewhere between the end points to indicate an intermediate rating.

When analyzing the results, marks on the left were scored as 0 , while marks on the right were scored as 100 . All statements referred to the patient's experience during the previous week. All 
participants completed the VAS form twice (time 1 and time 2) over the 3 days.

Other questionnaires filled in by patients were the THI questionnaire, the TFI questionnaire, the Beck Depression Inventory (BDI), and the Tinnitus and Hearing Survey (THS; with subscales $\mathrm{A}$ and $\mathrm{B})$. The THI is a self-reported measure consisting of 25 items divided into the following 3 subscales: functional (11 items), emotional (9 items), and catastrophic (5 items). Prefixes in the numbered questions (F, E, and C) indicate which subgroup the question comes from. There are 3 possible answers: "yes" (4 points), "sometimes" ( 2 points), and "no" (0 points). The total THI score ranges from 0 to 100 [Newman et al., 1996].

The TFI is the second most used questionnaire in the world. The TFI was created in 2012 and is a self-reporting measure used to evaluate tinnitus severity. It consists of 25 items divided into the following 8 subscales addressing different domains of tinnitus severity: intrusiveness ( 3 items), sense of control ( 3 items), cognition (3 items), sleep (3 items), audition (3 items), relaxation (3 items), quality of life ( 4 items), and emotions (3 items). The TFI uses a 10-point scale ranging from 0 to 10 , and all of the subscales contribute to a total score in ranging from 0 to 100 [Meikle et al., 2012]. The TFI is the only tinnitus questionnaire validated for sensitivity to changes in tinnitus-related distress [Fackrell et al., 2014; Baguley et al., 2016]. Both of the above questionnaires have been validated in Polish settings [Wrzosek et al., 2016; Skarzynski et al., 2017]. The BDI is a quick and easy-to-administer self-reporting measure commonly used for research or screening purposes [Beck et al., 1988; Zawadzki et al., 2009]. It consists of items relating to symptoms of depression such as hopelessness, irritability, cognitive feelings such as guilt, or feelings of being punished. It also covers physical symptoms such as fatigue and weight loss.

The THS questionnaire was created as a tool for determining how many complaints about tinnitus are due to hearing problems and how many are specifically due to tinnitus [Henry et al., 2015]. The instrument is composed of 3 parts. Section A (tinnitus) contains a list of 4 symptoms characteristic of tinnitus-related distress (difficulty sleeping, concentrating, or relaxing, and whether it is possible to focus attention away from the tinnitus) which are not caused by hearing loss. Section B (hearing) consists of 4 statements about hearing loss (problems with hearing in background noise, understanding speech, hearing soft voices, and hearing group conversations) which are not related to tinnitus. Section $C$ contains 2 items assessing the patient's problems with decreased sound tolerance. The THS questionnaire has also been validated for the Polish language [Raj-Koziak et al., 2017].

\section{Psychometric Evaluation of the VAS Ratings}

Correlations between Each VAS Rating

Assuming that the VAS ratings measure related constructs, correlations between the 4 ratings were calculated. We expected there might be moderate to strong correlations, justifying the recording of multiple VAS ratings. We did not expect to see extremely strong correlations, because that would mean that each of the 4 VAS ratings were measuring the same construct and therefore theoretically redundant.

\section{Quality Criteria}

Quality criteria for criterion validity, construct validity, reproducibility (agreement and reliability), and interpretability were de- rived from quality criteria used to measure the properties of health status questionnaires, as proposed by Terwee et al. [2007]. In detail, each of the criteria were derived as follows.

Criterion Validity. This refers to the extent to which VAS ratings relate to a gold standard. Henry et al. [2015] stated that "It is conceivable that the TFI will attain acceptance as a 'standard' tinnitus questionnaire, which would enable outcomes to be directly compared across different clinics and research studies". Based on the above finding and taking into account the fact that the TFI is the only tinnitus questionnaire validated for sensitivity to changes in tinnitus-related distress, it was regarded here as the "standard" tinnitus questionnaire. Terwee et al. [2007] give a positive rating if convincing arguments are presented that the standard being assessed is really "gold" and if the correlation with the gold standard is at least 0.70 .

Construct Validity. This refers to the extent to which the VAS scores relate to other instruments in a way that is consistent with a theoretically derived hypothesis. VAS scores were compared to the results of the THI questionnaire and the THS survey (subscale A), which measure annoyance as a handicap related to the tinnitus. We expected strong and moderate correlations (convergent validity) among the results of the THI and the THS survey (subscale A) and VAS. Correlation coefficient scores $>0.8$ were classified as "extremely strong," coefficients between 0.6 and 0.79 were classified as "strong," those between 0.3 and 0.59 were classified as "moderate," and those below 0.3 were classified as "weak" [Peterson, 1994; Fackrell et al. 2014].

Additionally, to investigate how the VAS-L measurement results are related to loudness measurements obtained with psychoacoustic matching procedures; psychoacoustic loudness of tinnitus was evaluated for the each patient. To identify tinnitus intensity (loudness) the examiner presented sounds that could possibly be similar to those described by the patient, among those emitted via audiometer at $10 \mathrm{~dB}$ HL above the hearing threshold. The loudness matching was indicated for the frequency of tinnitus indicated by the patient. Spearman's $\rho$ test was used to assess the results.

Discriminant validity was assessed comparing VAS measurement scores to the results of the THS (subscale B) questionnaire and the BDI. We expected relatively low correlations because these tools represent theoretically distinct constructs. Terwee et al. [2007] gives a positive rating for construct validity if hypotheses are specified in advance and at least $75 \%$ of the results are in correspondence with these hypotheses in a sample size of at least 50 patients.

The above mentioned criteria measuring criterion and construct validity cannot be fully applied to assess the possibility of coping with tinnitus because the THI, THS, and TFI questionnaires measure rather the handicap, annoyance, and tinnitus-related distress rather than the ability to cope with the problem. So, moderate correlations (convergent validity) among the results of VAS-C assessing the possibility of coping with tinnitus and the THI and the THS survey (subscale A) were expected.

Reproducibility

Reproducibility (agreement and reliability) concerns the degree to which repeated measurements (test-retest) provide similar answers. Terwee et al. [2007] did not rate the appropriateness of the time period of test-retest but only required its description and justification. 
Table 1. Spearman's correlation coefficients for scores on 3 VAS scales

\begin{tabular}{llll}
\hline & VAS-A & VAS-D & VAS-C \\
\hline VAS-L & 0.67 & 0.73 & 0.43 \\
VAS-A & & 0.79 & 0.47 \\
VAS-D & & & 0.54 \\
\hline
\end{tabular}

$p<0.01$ for all values.

Table 2. Spearman's correlation between VAS scores and those on the TFI, the THI, and the THS

\begin{tabular}{lllll}
\hline & VAS-L & VAS-A & VAS-D & VAS-C \\
\hline TFI & & & & \\
Intrusiveness & 0.75 & 0.67 & 0.69 & 0.49 \\
Sense of control & 0.54 & 0.66 & 0.65 & 0.54 \\
Cognition & 0.67 & 0.73 & 0.67 & 0.38 \\
Sleep & 0.65 & 0.67 & 0.69 & 0.29 \\
Auditory & 0.52 & 0.43 & 0.38 & 0.36 \\
Relaxation & 0.61 & 0.72 & 0.68 & 0.49 \\
Quality of life & 0.58 & 0.76 & 0.62 & 0.41 \\
Emotional & 0.63 & 0.83 & 0.79 & 0.56 \\
\hline Total score & 0.73 & 0.81 & 0.77 & 0.52 \\
\hline THI & & & & \\
Functional & 0.68 & 0.76 & 0.73 & 0.47 \\
Emotional & 0.58 & 0.83 & 0.76 & 0.50 \\
Catastrophic & 0.63 & 0.76 & 0.70 & 0.54 \\
\hline Total score & 0.67 & 0.83 & 0.78 & 0.52 \\
\hline THS & & & & \\
Tinnitus & 0.60 & 0.75 & 0.69 & 0.41 \\
Hearing & 0.40 & 0.40 & 0.22 & 0.34 \\
\hline
\end{tabular}

$p<0.01$ for all values.

Agreement concerns the absolute measurement error and reliability concerns the degree to which patients can be distinguished from each other, despite measurement error. Bland-Altman limits of agreement were calculated as the mean difference in scores between repeated measurements (mean change) $\pm 1.96 \times$ SD of these differences (SD change). A result of $95 \%$ agreement was treated as an indication of high agreement [Bland and Altman, 1986].

The reliability of the THS questionnaire was assessed using intraclass correlations (ICC), with scores $>0.70$ indicating a high reliability.

\section{Interpretability}

Interpretability is defined as the degree to which one can assign qualitative meaning to quantitative scores. In our study we tried to provide information about what score of VAS scales would be clinically meaningful (i.e., provide information about tinnitus severity and determine whether a tinnitus-specific clinical service is required). We tried to find the values of VAS at which tinnitus severity becomes clinically relevant to patients. For this purpose, the receiver operating characteristic method was used. It plays an important role in evaluation of the diagnostic ability of the test to discriminate the true state of the subjects and find the optimal cutoff values. The receiver operating characteristic curve is obtained by plotting at different cut-off and it combines information on sensitivity (true-positive rate) and specificity (true-negative rate), and the area under curve (AUC) is treated as a measure of accuracy. Kleinbaum and Klein [2010] criteria were used to assess the results of AUC values. Scores between 0.5 and 0.6 was classified as "failed," coefficients between 0.6 and 0.7 were classified as "poor," those between 0.7 and 0.8 were classified as "fair," those between 0.8 and 0.9 as were classified "good," and those between 0.9 and 1.0 were classified as "excellent" discrimination.

As the external criterion (anchor), the results of the TFI and the results of the THI were used. TFI questionnaire scores above about 50 are likely to indicate severe problems with tinnitus and these patients require referral to specialty tinnitus care; therefore, TFI values above 50 were taken as the criterion of tinnitus severity [Meikle et al., 2012]. THI questionnaire scores above 56 according to criteria by McCombe et al. [2001] were taken as another criterion of tinnitus severity.

\section{Results}

\section{Correlations between VAS Results}

Spearman's correlation coefficient score results among VAS were moderate to strong. The results are shown in Table 1.

\section{Criterion Validity}

Spearman's correlations coefficients between VAS scores and TFI global scores were: VAS-L, $\rho=0.73$; VASA, $\rho=0.81$; VAS-D, $\rho=0.77$; and VAS-C, $\rho=0.52$. The results are shown in Table 2. According to the criteria of Terwee et al. [2007], the VAS scale measures received a positive rating for criterion validity based on comparisons with TFI global scores, with the exception of VAS-C.

\section{Construct Validity}

Spearman's correlation coefficients between scores on the VAS and the THI global score were: VAS-L, $\rho=0.67$; VAS-A, $\rho=0.83$; VAS-D, $\rho=0.78$; and VAS-C, $\rho=0.52$. Table 2 shows the results, including those for the 3 subscales of the THI questionnaire (functional, emotional, and catastrophic).

Correlations between the VAS scores and the THI results were strong for VAS-L and VAS-D, extremely strong for VAS-A, and moderate for VAS-C. These results received a positive rating based on the criteria of Terwee et al. [2007]. Convergent validity between VAS-L, VAS-A, 


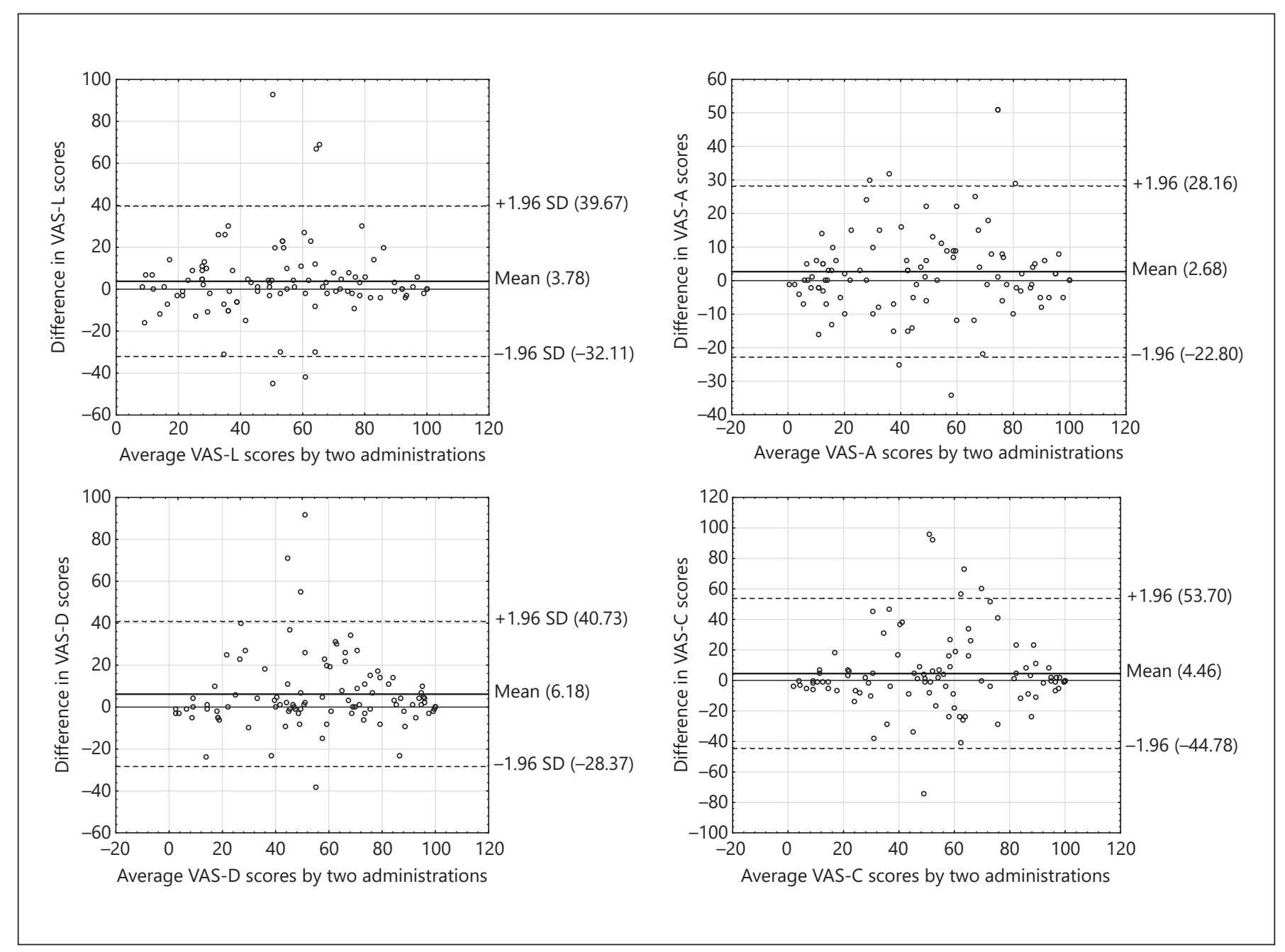

Fig. 2. Bland-Altman plots of test-retest agreement for repeated measures of VAS scores (VAS-L, VAS-A, VAS$\mathrm{D}$, and VAS-C). The dashed lines indicate $95 \%$ limits of agreement; the solid line marks the mean difference.

VAS-D, and VAS-C, and subscale A of the THS questionnaire amounted to $0.60,0.75,0.69$, and 0.41 , respectively. Correlations were strong for VAS-L, VAS-A, and VAS-D and moderate for VAS-C.

Convergent validity results between the VAS scales and subscale A of the THS questionnaire are shown in Table 2 . Results were generally strong (75\% of results) or moderate (25\% of results) and received a positive rating according to the criteria of Terwee et al. [2007] (i.e., the results are consistent with the assumed hypothesis).

Spearman's correlation between VAS-L and loudness measures obtained with psychoacoustic matching procedures amounted to $0.28(p<0.01)$.

Divergent validity between VAS scores and answers on the THS (subscale B) questionnaire received a positive rating based on the criteria of Terwee et al. [2007]. Correlations were weak or moderate, from which it can be concluded that the results are consistent with the assumed hypothesis.

Divergent validity between VAS scores and BDI amounted to 0.43 for VAS-L (moderate correlation), 0.68 for VAS-A (strong), 0.52 for VAS-D (moderate), and 0.42 for VAS-C (moderate). Convergent validity between VAS scales and BDI was $75 \%$, consistent with the assumed hypothesis.

\section{Reproducibility}

The identified limits of agreement for were 95\% for VAS-L, $93 \%$ for VAS-A, $96 \%$ for VAS-D, and $95 \%$ for VAS-C (Fig. 2). The Bland-Altman limits of agreement 
Table 3. Limits of agreement for VAS-L, VAS-A, VAS-D, and VAS-C

\begin{tabular}{|c|c|c|c|c|c|c|}
\hline & Test & Retest & Mean change & SD change & Limits of agreement & Agreement, \% \\
\hline VAS-L & $55.47(27.30)$ & $51.69(26.42)$ & 3.78 & 18.31 & -32.11 to +39.67 & 95 \\
\hline VAS-A & $49.19(31.25)$ & $46.51(30.10)$ & 2.68 & 13.00 & -22.80 to +28.16 & 93 \\
\hline VAS-D & $58.99(28.97)$ & $52.01(28.98)$ & 6.18 & 17.63 & -28.37 to +40.73 & 96 \\
\hline VAS-C & $56.47(31.66)$ & $52.01(30.93)$ & 4.46 & 25.12 & -44.78 to +53.70 & 94 \\
\hline
\end{tabular}

Table 4. Cut-off points and corresponding AUC for 4 VAS

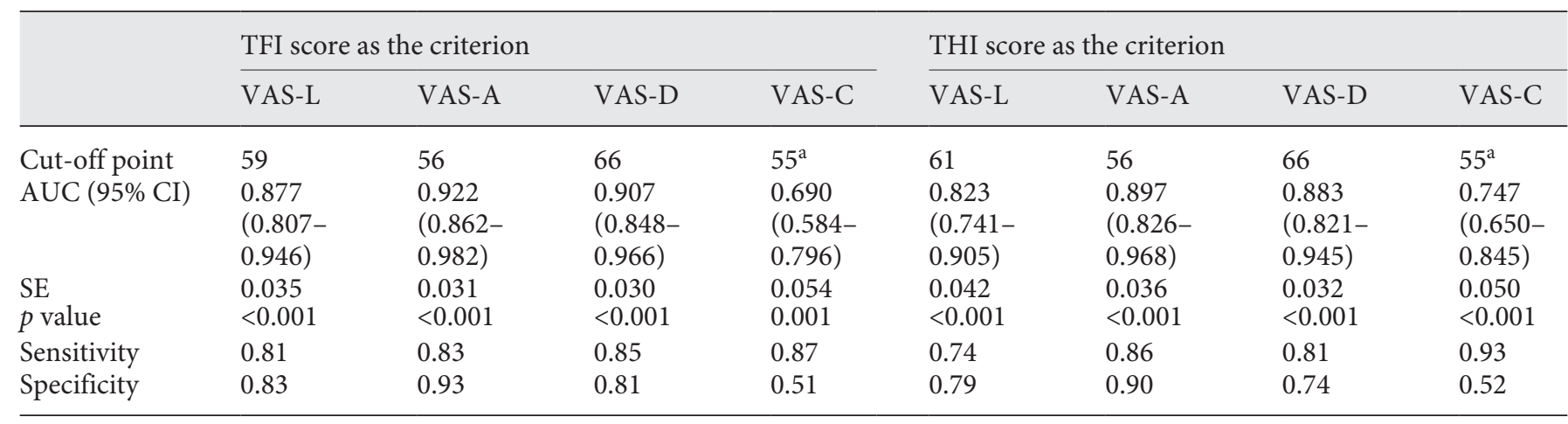

${ }^{a}$ Values less than that listed indicate a higher tinnitus severity.

are shown in Table 3. Agreement between VAS-L and VAS-D received a positive rating, with scores of 95\%; however, results for VAS-A (93\%) and VAS-C (94\%) were just below those required for a positive rating.

ICC values for single measurements were 0.76 for VAS-L, 0.90 for VAS-A, 0.80 for VAS-D, and 0.67 for VAS-C. ICC values $>0.70$ indicated that the first $3 \mathrm{had}$ a high reliability but VAS-C was just below this threshold.

\section{Interpretability}

AUC values for selected cut-offs are shown in Table 4. In assessing the AUC values, the recommendations of Kleinbaum and Klein [2010] suggest that, based on the results of the TFI questionnaire, the criteria for VAS-L should be classified as good and excellent for VAS-A and VAS-D and poor for VAS-C. The corresponding cut-off points for VAS scales were 59 for VAS-L, 56 for VAS-A, 66 for VAS-D, and 55 for VAS-C.

Based on the results of the THI questionnaire, AUC values were good for VAS-L, VAS-A, and VAS-D and fair for VAS-C. The corresponding cut-off points were 61 for VAS-L, 56 for VAS-A, 66 for VAS-D, and 55 for VAS-C.

\section{Discussion}

The analyses used in this study aimed to confirm the construct validity, criterion validity, and reliability of 4 VAS scales designed to measure tinnitus loudness, annoyance and distress, and how well the patient was coping. The results also provide criteria for tinnitus severity from which certain scores on VAS-L, VAS-A, VAS-D, and VAS-C may raise concern clinically.

As expected, moderate and strong positive correlations were found among the 4 VAS scales. An extremely strong correlation would have indicated redundancy, meaning that 1 , not 4 , scales would need to be marked. Our results point to each VAS scale being different, indicating that each scale identifies a different aspect of tinnitus.

Terwee et al. [2007] gives a positive rating for criterion validity if the correlation with the gold standard is at least 0.70 . In our study, the TFI questionnaire was used as the standard. Some justification for using this questionnaire was provided in Materials and Methods, but in addition we note that Fackrell et al. [2014] found that the TFI met 8 of the 10 criteria for good tinnitus measurement tools, making it the best questionnaire. The 3 VAS scales for tin- 
nitus loudness, annoyance, and distress received positive ratings for content validity. However, the VAS-C scale, which measures how well the patient is coping with tinnitus, received a negative rating. As described in Materials and Methods, criteria for validity cannot be properly applied to assess how well a patient is coping with tinnitus because the TFI questionnaire has only 1 subscale (sense of control) related to coping with tinnitus. A recent review of tinnitus coping strategies revealed deficiencies in current research dedicated to the issue [Martz and Henry, 2016]. It may be that the moderate correlation between VAS-C and TFI was due to the general lack of tools for measuring how well a person is coping with tinnitus. To assess the content validity of VAS-C, a psychometrically robust instrument dedicated specifically to measuring this theoretical construct is needed [Martz and Henry, 2016].

There was convergent validity between the VAS scales and the THI questionnaire, and the THS (subscale A) received a positive rating according to the criteria of Terwee et al. [2007] because $75 \%$ of the results were consistent with the assumed hypothesis. Our results are comparable to those of other studies. Fiqueiredo et al. [2009] found significant Spearman's correlations between a VAS scale measuring tinnitus volume/disturbance and the THI ( $r=$ 0.564, $p<0.001, n=43$ ). Adamchic et al. [2012] found strong correlations between both VAS-L and VAS-A (baseline values) and the Tinnitus Questionnaire, with values of $0.4-0.56$.

As mentioned above, the criteria for assessing content validity and construct validity cannot be properly applied to gauge how well the person is coping with tinnitus because the THI, the THS, and the TFI measure annoyance and distress rather than the degree of handicap in coping with the problem. This would explain the lower correlation between the results of VAS-C (how well the person is coping with tinnitus) and those of the THI and the THS (subscale A).

We obtained only a weak correlation between VAS-L and loudness measurements made by psychoacoustic matching. The weak correlation confirms that these measurements are related in some way; however, it is unclear which method provides data of higher validity and relevance [Adamchic et al., 2012]. The results of our further study [Raj-Koziak, in press], examining the relationship between psychoacoustic matches of tinnitus loudness and tinnitus loudness measured with a VAS, revealed that only in normal hearing patients was the correlation moderate and statistically significant.

Discriminant validity between the VAS scales and the THS (subscale B) showed weak and moderate correla- tions, implying that the results are in accordance with the hypothesis. Both results received a positive rating using the criteria suggested by Terwee et al. [2007]. Moderate and strong correlations between the VAS scales and the $\mathrm{BDI}$, which is a tool representing theoretically distinct constructs, may be due to the fact that tinnitus in some cases can be accompanied by depression.

The time between test and retest of the VAS scales was 3 days. Choosing such a short test-retest period was a question of practicality, economics, and the certainty of tinnitus stability. There are also promising results from reports on health-related instruments measuring test-retest stability, where there were no difference in the results of 2-day and 2-week test-retest intervals [Marx et al., 2003]. The disadvantage of using such a short time between test and retest for THS assessment is that people are likely to remember their answers from the first test and tend to give the same answers again. ICC results with scores $>0.70$ indicates a high reliability for VAS-L, VAS$\mathrm{A}$, and VAS-D. The ICC of the VAS-C result (i.e., 0.67) was close to that for high reliability.

The reproducibility of the VAS was evaluated using the Bland-Altman method of limits of agreement and the results indicated that VAS-L and VAS-D were in high agreement (95\%) and that VAS-A and VAS-C were very close to meeting the quality criteria proposed for health status questionnaires.

VAS scales are attractive tools because they are simple and quick to administer, and they have been suggested as useful for introductory assessments [Torrance et al., 2001]. The cutoff points determined here by the VAS method open up the possibility that tinnitus severity could be quickly indicated in this way, allowing clinicians to make a decision about whether a patient should be referred for tinnitus-specific intervention.

The results of correlation between VAS-L, VAS-A, and VAS-D were consistent, which means that patients had similar marks on the scales. Correlations with the VAS-C scale were also consistent but weaker. This could reflect the patients' individual abilities to cope with tinnitus.

Scores of VAS-L, VAS-A, and VAS-D exceeding 60, 56 , and 56, respectively, and results of VAS-C below 55 are clinically meaning full, provide information about troublesome tinnitus, and determine that a tinnitus-specific clinical service is required. Patients with low abilities to cope with tinnitus (VAS-C values $<55$ ) should be also referred to a psychologist.

Because tinnitus is multidimensional and subjective, it is not possible to obtain objective measures. The establishment of normative standards would enable tinnitus 
psychoacoustics measures to be evaluated with respect to a set of norms [Henry, 2016]. The results of the present study confirm that VAS may be used as a reliable tool for making valid measurements of tinnitus loudness, annoyance, distress, and how well the patient is coping. It could play a role in obtaining quick information about a tinnitus patient at their first intake (while recognizing the problems mentioned above). During the first visit to the ENT office, the aim is to make a diagnosis about the organic cause of the condition, which could be otosclerosis, Meniere's disease, chronic otitis media, vestibular schwannoma, or something else. Using a VAS at this stage of the diagnosis could work as a fast counselling tool and one which is easy to perform and understand and gives quick information about the level of tinnitus. A result on VAS exceeded at least on one scale indicates a significant problem and requires reference to tinnitus-specific intervention. VAS are not stand-alone tools and will never replace the use of a full questionnaire. On the contrary, we suggest that VAS and questionnaires can complement each other, that VAS should be used as a screening tool, and that THI and TFI questionnaires should be used to measure tinnitus handicap and for outcome measures [Landgrebe et al., 2012; Fackrell et al., 2014].

\section{Limitations of This Study}

The population of people with tinnitus who were hospitalized may not be representative of the general tinnitus population.
To assess the validity of VAS-C it would be advisable to use psychometrically robust instruments designed specifically to measure this theoretical construct.

\section{Conclusions}

VAS scales are valid and reliable brief screening tools which make it possible to obtain quick information about tinnitus at the patient's first intake. The cutoff points which we have determined can be used as criteria for tinnitus severity and they can help in making a decision about whether further intervention is needed.

\section{Acknowledgement}

The authors express gratitude to colleagues from the World Hearing Center - Katarzyna Bienkowska, Beata Dziendziel, Justyna Kutyba, and Karolina Penar - for their help in collecting data.

\section{Disclosure Statement}

The authors declare that there is no conflict of interests.

\section{Funding Sources}

This research received no specific grant from any funding agency in the public, commercial, or not-for-profit sectors.

\section{References}

Adamchic I, Langguth B, Hauptmann C, Tass PA: Psychometric evaluation of visual analog scale for the assessment of chronic tinnitus. Am J Audiol 2012;21:215-225.

Axelsson A, Ringdahl A: Tinnitus: a study of its prevalence and characteristics. Br J Audiol 1989;23:53-62.

Baguley DM, Cope TE, McFerran DJ: Functional auditory disorders. Handb Clin Neurol 2016; 139:367-378.

Beck AT, Steer RA, Carbin MG: Psychometric properties of the Beck Depression Inventory: twenty-five years of evaluation. Clin Psychol Rev 1988;8:77-100.

Bland JM, Altman DG: Statistical methods for assessing agreement between two methods of clinical measurement. Lancet 1986;1:307310 .
Davis A; MRC Institute of Hearing Research: Hearing in adults: the prevalence and distribution of hearing impairment and reported hearing disability in the MRC Institute of Hearing Research's National Study of Hearing. London, Whurr, 1995.

Fabijańska A, Smurzyński J, Hatzopoulos S, Kochanek K, Bartnik G, Raj-Koziak D, et al: The relationship between distortion product otoacoustic emissions and extended high-frequency audiometry in tinnitus patients. 1 . Normally hearing patients with unilateral tinnitus. Med Sci Monit 2012;18:765-770.

Fackrell K, Hall D, Barry J, Hoare D: Tools for tinnitus measurement: development and validity of questionnaires to assess handicap and treatment effects; in Signorelli F, Turjman F (eds): Tinnitus: Causes Treatment and Short and Long-Term Health Effects. New York, Nova Science, 2014, pp 13-60.
Figueiredo RR, Azevedo AA, Oliveira P de M: Correlation analysis of the visual-analogue scale and the Tinnitus Handicap Inventory in tinnitus patients. Braz J Otorhinolaryngol 2009;75:76-79.

Henry JA: Measurement of tinnitus. Otol and Neurotol 2016; 37:276-286.

Henry JA, Griest S, Zaugg TL, Thielman E, Kaelin $\mathrm{C}$, Galvez $\mathrm{G}$, et al: Tinnitus and hearing survey: a screening tool to differentiate bothersome tinnitus from hearing difficulties. Am J Audiol 2015;24:66-77.

Kim H-J, Lee H-J, An S-Y, Sim S, Park B, Kim SW, et al: Analysis of the prevalence and associated risk factors of tinnitus in adults. PLoS One 2015;10:e0127578.

Kleinbaum DG, Klein M: Logistic Regression: A Self-Learning Text. Berlin, Springer, 2010. 
Landgrebe M, Azevedo A, Baguley D, Bauer C, Cacace A, Coelho C, et al: Methodological aspects of clinical trials in tinnitus: a proposal for an international standard. J Psychosom Res 2012;73:112-121.

Langguth B, Searchfield GD, Biesinger E, Greimel $\mathrm{KV}$ : History and questionnaires; in Møller AG, Langguth B, DeRidder D, Kleinjung T: Textbook of Tinnitus. New York, Springer, 2011, pp 387-404.

Martinez C, Wallenhorst C, McFerran D, Hall DA: Incidence rates of clinically significant tinnitus: 10-year trend from a cohort study in England. Ear Hear 2015;36:e69-e75.

Martz E, Henry JA: Coping with tinnitus. J Rehabil Res Dev 2016;53:729-742.

Marx RG, Menezes A, Horovitz L, Jones EC, Warren RF: A comparison of two time intervals for test-retest reliability of health status instruments. J Clin Epidemiol 2003;56:730735.

McCombe A, Baguley D, Coles R, McKenna L, McKinney C, Windle-Taylor P, et al: Guidelines for the grading of tinnitus severity: the results of a working group commissioned by the British Association of Otolaryngologists, Head and Neck Surgeons, 1999. Clin Otolaryngol Allied Sci 2001;26:388-393.
McCormack A, Edmondson-Jones M, Fortnum $\mathrm{H}$, Dawes P, Middleton H, Munro KJ, et al: The prevalence of tinnitus and the relationship with neuroticism in a middle-aged UK population. J Psychosom Res 2014;76:56-60.

Meikle MB, Henry JA, Griest SE, Stewart BJ, Abrams HB, McArdle R, et al: The Tinnitus Functional Index: development of a new clinical measure for chronic, intrusive tinnitus. Ear Hear 2012;33:153-176.

Newman CW, Jacobson GP, Spitzer JB: Development of the Tinnitus Handicap Inventory. Arch Otolaryngol Head Neck Surg 1996;122: 143-148.

Peterson RA: Meta-analysis of Cronbach's coefficient alpha. $1994 \mathrm{https} / /$ academic.oup. $\mathrm{com} / \mathrm{jcr} / \mathrm{article}-\mathrm{abstract} / 21 / 2 / 381 /$ 1799516/A-Meta-analysis-of-Cronbach-sCoefficient-Alpha?redirectedFrom $=$ fulltext (accessed September 12, 2017).

Raj-Koziak D, Gos E, Rajchel JJ, Piłka A, Skarżyński H, Rostkowska J, et al: Tinnitus and Hearing Survey: a Polish study of validity and reliability in a clinical population. Audiol Neurotol 2017;22:197-204.

Ries PW: Prevalence and characteristics of persons with hearing trouble: United States, 1990-91. Vital Health Stat 10 1994;1-75.
Shargorodsky J, Curhan GC, Farwell WR: Prevalence and characteristics of tinnitus among US adults. Am J Med 2010;123:711-718.

Skarzynski PH, Raj-Koziak D, J Rajchel J, Pilka A, Wlodarczyk AW, Skarzynski H: Adaptation of the Tinnitus Handicap Inventory into Polish and its testing on a clinical population of tinnitus sufferers. Int J Audiol 2017;56:711715

Terwee CB, Bot SDM, de Boer MR, van der Windt DA, Knol DL, Dekker J, et al: Quality criteria were proposed for measurement properties of health status questionnaires. J Clin Epidemiol 2007;60:34-42.

Torrance GW, Feeny D, Furlong W: Visual analog scales: do they have a role in the measurement of preferences for health states? Med Decis Making 2001;21:329-334.

Wrzosek M, Szymiec E, Klemens W, Kotyło P, Schlee W, Modrzyńska M, et al: Polish translation and validation of the Tinnitus Handicap Inventory and the Tinnitus Functional Index. Front Psychol 2016;7:1871.

Zawadzki B, Popiel A, Pragłowska E: Charakterystyka psychometryczna polskiej adaptacji Kwestionariusza Depresji BDI-II Aarona T. Becka. Psychol Etol Genet 2009;19:71-95. 